\title{
Teditorial
}

\section{Prevention of self-harm and suicide in adolescents}

\author{
Erita Istriana \\ Department of Psychiatry Faculty of Medicine Trisakti University
}

\begin{abstract}
Adolescence is a transitional stage in physical and psychological development, and in this stage multiple physical, emotional and social changes, can make adolescents vulnerable to mental health problems like self-harm and suicide. Self-harm and suicide are serious problems in children, adolescents and young people with the highest rates seen in those aged 16-24 years, ${ }^{(1)}$ and self-harm is highly correlated with the presence of anxiety disorders and depression. ${ }^{(2)}$
\end{abstract}

The term self-harm can be used to refer to acts of self-poisoning or self-injury (eg, cutting, scratching, breaking bones and burning) carried out intentionally regardless of motive or suicidal intent. ${ }^{(3)}$ There is a strong association between self-harm and risk of future suicide, with approximately $50 \%$ of adolescents who die by suicide having previously self-harmed, and selfharm increasing the risk of death by suicide approximately tenfold. ${ }^{(4)}$ Predicting suicide and repetition of selfharm in adolescents is a challenge as it is usually a secretive and hidden behaviour The most common reasons why adolescents participate in self-injury are as a coping mechanism, a means of relief, for the regulation of feelings, self-punishment, attention seeking and sensation seeking. ${ }^{(5)}$ There is growing evidence that perfectionism and selfcriticism, low self-esteem, social isolation, impulsivity, hopelessness, poor parent-child attachment, and exposure to negative life events are associated with self-harm in clinical and community populations. ${ }^{(5,6)}$ But only a small proportion of individuals who self-harm present to hospitals, meaning that this behaviour is largely hidden (at least from clinical services) at the community level.

Given the heterogeneity and complexity of pathways deaths to suicide, an approach that includes effective universal, secondary, and tertiary prevention strategies will likely yield the greatest benefits at a population level. For tertiary prevention among adolescents who have already shown self-harm behavior or tendencies, intensive community treatment strategies that reduce unnecessary exposure to hospitalization and crisis services may be an important component. ${ }^{(6)}$

As a place where many young people spend a considerable proportion of their time, schools are one of the most important institutions that are in a position to help-self injurers, and are logical and natural settings for youth or adolescents suicide prevention efforts. ${ }^{(7)}$ Suicide prevention has not typically been a priority for schools unless there has been a suicide death. The programmes which have aim of enhancing emotional wellbeing among a broader population of students by using a variety of mental health promotion and mental illness prevention activities are important to cultivate the development of a set of protective factors. School-based programme can be considered to have a role to play in youth or adolescents suicide prevention strategies by using surveillance and instructional methods to enhance the identification and referral, through many activities that should take account of the particular characteristics of adolescents suicide and self-harm (for example, target issues around the social transmission of suicide and self-harm, sexual orientation and bullying in young people, promote help-seeking behaviour, and foster selfesteem and resilience), and also improve the distress-coping skills, of students who may be at risk for suicide. ${ }^{(7,8)}$

Study by Wyman et al. ${ }^{(9)}$ also showed that strengthening social integration could prevent suicidal behavior and have implications for developing network-informed suicide prevention (NISP) approaches in schools and other education settings. Network-informed suicide prevention interventions will involve youth and adults and is likely to most be effective and safe by incorporating multiple focuses: maximizing 
protective bonds across school populations, increasing opportunities for group cohesion including integrated youth-adult networks, and promoting influence of youth with healthy coping. ${ }^{(9)}$

The identification of successful prevention initiatives aimed at adolescents and those at specially high risk, and the establishment of effective treatments for those who self-harm, are also paramount needs. In addition the presence of social support such support includes good peer relations, support from teachers, and a warm and stable relationship with their parents, also plays an especially important protective role to prevent self-harm and suicidal behavior among adolescents.

\section{REFERENCES}

1. Chan MKY, Bhatti H, Meader $\mathrm{N}$, et al. Predicting suicide following self-harm: systematic review of risk factors and risk scales. Br J Psychiatry 2016;209:277-83.

2. Mars B, Heron J, Crane C, et al. Differences in risk factors for self-harm with and without suicidal intent: findings from the ALSPAC cohort. J Affect Disord 2014;168:407-14. doi: 10.1016/j.jad.2014.07.009. Hawton K, Bergen H, Kapur N, et al. Repetition of self-harm and suicide following self-harm in children and adolescents: findings from the multicentre study of self-harm in England. J Child Psychol Psychiatry 2012;53:1212-9.

3. Harris IM, Beese S, Moore D. Predicting future self-harm or suicide in adolescents: a systematic review of risk assessment scales/tools. BMJ Open 2019;9:e029311. doi:10.1136/bmjopen2019-029311.

4. Yakeley J, James WB. Psychodynamic approaches to suicide and self-harm. B J Psych Advances 2018, vol. 24, 37-45 doi: 10.1192/bja. 2017.6.

5. Asarnow JR, Mehlum L. Practitioner review: treatment for suicidal and selfharming adolescents-advances in suicide prevention. J Child Psychol Psychiatry 2019, 60:10, pp 10461054 doi:10.1111/jepp.13130.

6. Singer JB, Erbacher TA, Rosen P. School-based suicide prevention: a framework for evidence-based practice. School Mental Health 2019;11;54-71. https://doi.org/10.1007/s12310018-9245-8.

7. Hawton K, Saunders KEA, O'Connor RC. Selfharm and suicide in adolescents. Lancet 2012;379:2373-82. doi: 10.1016/S0140-6736(12) 60322-5.

8. Wyman PA, Pickering TA, Pisani AR, et al. Peer-adult network structure and suicide attempts in 38 high schools: implications for network-informed suicide prevention. Journal of Child Psychology and Psychiatry 2019,60:10, pp 1065-1075 doi:10.1111/jcpp.13102. 